\title{
Clozapine-related Paroxysmal Supraventricular Tachycardia: a Case Report
}

\author{
SJ Kim, MS Gim
}

\begin{abstract}
Clozapine is considered to be more effective than other antipsychotic drugs for treating treatmentresistant schizophrenia. However, side effects of clozapine include agranulocytosis and, less commonly, cardiovascular disease, which is occasionally fatal. We describe a 56-year-old woman who developed clozapine-related paroxysmal supraventricular tachycardia during clozapine dose titration and had a recurrence despite being treated with verapamil. For treatment-resistant schizophrenia, a slow titration of the clozapine dose is necessary, and potential cardiac side-effects should be monitored.
\end{abstract}

Key words: Clozapine; Schizophrenia; Tachycardia, supraventricular; Verapamil

Dr Su-Jin Kim, MD, Department of Psychiatry, College of Medicine, Inje University, Sanggye Paik Hospital, Seoul, Korea

Dr Min-Sook Gim, MD, PhD, Department of Psychiatry, College of Medicine, Inje University, Sanggye Paik Hospital, Seoul, Korea

Address for correspondence: Dr Min-Sook Gim, MD, PhD, Department of Psychiatry, College of Medicine, Inje University, 1342 Dongilro, Nowon-gu, Seoul, 01757, Korea.

Tel: +82.2-950-1082; Fax: +82.2-936-8069; Email: numbers@paik.ac.kr

Submitted: 24 July 2017; Accepted: 12 December 2017

\section{Introduction}

Clozapine is a second-generation antipsychotic agent, which is considered to be more effective than other antipsychotics against treatment-resistant schizophrenia. However, clozapine's use is restricted because of side-effects such as agranulocytosis. ${ }^{1}$ Clozapine is also associated with increased risk of seizure ${ }^{2}$ and cardiac side-effects such as sinus tachycardia, orthostatic hypotension, and prolongation of the corrected QT (QTc) interval. Rare side-effects include myocarditis, cardiomyopathy, ${ }^{3}$ and sudden cardiac death. The mechanism of clozapine-induced cardiac death remains unclear; however, it likely involves ventricular arrhythmia, related to blocking of potassium channels by clozapine, leading to prolonged cardiac repolarisation. ${ }^{4}$ Nonetheless, clozapine-induced cardiovascular disease is of low prevalence.

Paroxysmal supraventricular tachycardia (PSVT) includes atrioventricular nodal re-entrant tachycardianamely, atrioventricular re-entry with an accessory pathway - and atrial tachycardia. Among clozapine-treated patients, the prevalence of PSVT is 2.25 per 1000 persons. The risk for females is 2 times as high as that for males. The risk of PSVT for adults aged 65 years and older is 5 times as high as that for people younger than 65 years. ${ }^{5} \mathrm{PSVT}$ affects quality of life but is rarely life threatening. ${ }^{6}$ Its prognosis and treatment vary considerably depending on the mechanism and underlying cardiac problems. Vagal manoeuvres, beta-blockers, verapamil, and diltiazem are used to end acute episodes of PSVT and to reduce the frequency of chronic episodes. Catheter ablation should be considered if antiarrhythmic drugs are ineffective. Electrophysiology studies are necessary for risk evaluation of those working in certain occupations. However, routine follow-up without therapy is acceptable in some patients.

Here we describe a 56-year-old woman who developed clozapine-related PSVT during clozapine dose titration and had recurrence despite being treated with verapamil.

\section{Case Report}

In June 2015 a 56-year-old woman was admitted to the psychiatry unit of Sanggye Paik Hospital, Korea, with delusions of persecution and auditory hallucinations. We diagnosed her as having an acute episode of schizophrenia. Five years before this admission, she separated from her husband and had delusions that her husband tapped her telephone and watched her. Eight months before this admission, she was admitted to our psychiatry unit and treated with risperidone $(4 \mathrm{mg} /$ day $)$ and lorazepam $(0.5 \mathrm{mg} / \mathrm{day})$. Her symptoms resolved and she was discharged from hospital after 1 month. She later restored good relations with her husband. Because of excessive daytime sleepiness and muscle rigidity, she reduced her antipsychotic drug dosage and then discontinued medication. About 1 month later, she moved and visited another clinic and was prescribed risperidone $(0.5 \mathrm{mg} / \mathrm{day})$, lorazepam $(1 \mathrm{mg} / \mathrm{day})$, and alprazolam $(0.5 \mathrm{mg} / \mathrm{day})$.

Approximately 3 months before this admission, she was readmitted with delusions of persecution, auditory 
hallucinations, and irritability. To avoid resistant to risperidone, she was treated with long-acting injection of paliperidone (12 mg/day). One month later, she was discharged home with paliperidone (12 mg/day), benztropine ( $1 \mathrm{mg} /$ day $)$, lorazepam $(0.5 \mathrm{mg} /$ day $)$ and was followed up in an out-patient clinic. However, her sleep pattern became irregular because she needed to care for her husband whose finger had been accidentally amputated.

One month before this admission, she was readmitted with recurrence of psychotic symptoms. She was first treated with olanzapine titrated up to a dose of $25 \mathrm{mg} /$ day. However, symptoms did not improve; hence, olanzapine was cross-tapered and amisulpride was titrated up to a dose of $1200 \mathrm{mg} /$ day. Nonetheless, her psychotic symptoms worsened and she developed extrapyramidal symptoms such as muscle rigidity. Her electrocardiogram (ECG) showed QTc prolongation. Her psychotic symptoms remained unresolved by treatment with risperidone, paliperidone, olanzapine, and amisulpride. Thus, we prescribed clozapine for treatment-resistant schizophrenia. Before administering clozapine, we performed baseline tests, including a complete blood cell count with differential white blood cell count, lipid profile, liver function, renal function, electrolytes, fasting glucose, thyroid function, haemoglobin A1c, urinalysis, ECG, electroencephalogram, and chest X-ray. Two days before treatment started, her QTc interval was $478 \mathrm{~ms}$, which indicated borderline QTc prolongation. On the day of treatment, her QTc interval was $444 \mathrm{~ms}$, which was within the normal range (<470 ms for female). The dose of clozapine was started at $25.0 \mathrm{mg}$, followed by daily increases of $12.5 \mathrm{mg}$ since day 3. The dose of amisulpride was gradually tapered. For her sleep disorder, trazodone $(50 \mathrm{mg} /$ day $)$ and lorazepam (1 mg/day) were prescribed. On day 9 of the titration, the clozapine dose reached $150 \mathrm{mg} /$ day, together with amisulpride $200 \mathrm{mg} /$ day, trazodone $50 \mathrm{mg} /$ day, and lorazepam $1 \mathrm{mg} /$ day.

The patient did not complain of any symptoms; however, a routine check of her vital signs revealed low blood pressure and tachycardia (160-230 beats/min). We suspected PSVT and injected adenosine $(6 \mathrm{mg})$. Verapamil was started at a dose of $180 \mathrm{mg} /$ day. We stopped the amisulpride and temporarily halted the increase of the clozapine dose. Clozapine therapy was continued at 150 $\mathrm{mg}$ /day. However, her psychotic symptoms persisted, and we decided to slowly increase the clozapine dose. On day 17 , her psychotic symptoms improved. The final dose of clozapine was $250 \mathrm{mg} /$ day, together with trazodone 50 $\mathrm{mg}$ /day, lorazepam $1 \mathrm{mg} /$ day, and verapamil $180 \mathrm{mg} /$ day. On day 18 , the patient complained of dizziness in a supine position and her blood pressure was $60 / 40 \mathrm{~mm} \mathrm{Hg}$. Her heart rate was 167 beats/min, which suggested recurrence of PSVT. Adenosine (6 mg) was injected (with direct current cardioversion standby) and her blood pressure and heart rate recovered. Verapamil (180 $\mathrm{mg}$ ) and clozapine $(250 \mathrm{mg}$ ) were thus continued. On day 26 (day 71 of admission), her psychotic symptoms resolved and she was discharged with follow-up care at an outpatient clinic. An electrophysiology study was proposed to determine the mechanism of PSVT; however, the patient declined it and the cardiologist believed that it was unnecessary.

\section{Discussion}

The occurrence of temporal PSVT in our patient was likely associated with clozapine titration. Clozapine has been reported to affect cardiac electrical conduction. The vagolytic effects of clozapine and its effects on calcium channels and repolarisation might provoke PSVT. ${ }^{8,9}$ Although PSVT is not fatal, treatment with clozapine can result in fatal ventricular tachycardia. In particular, QTc prolongation increases the risk of arrhythmia. ${ }^{10}$ Thus, although clozapine is rarely associated with myocarditis and cardiomyopathy, it is important to assess and correct QTc prolongation before clozapine titration. ${ }^{11}$ To reduce the risk of clozapine-induced cardiac side-effects, medications associated with QTc prolongation should be withdrawn before starting clozapine. In addition, ECG should be regularly monitored, and clinical symptoms suggestive of cardiac problems (eg, dyspnoea, chest discomfort, fatigue, and influenza-like symptoms) should be closely observed. Cardiac markers (serum troponin and creatine kinase-MB) should also be assessed.

We suggest (1) avoiding medications that cause QTc prolongation, (2) regular monitoring of vital signs and ECG, (3) slowly titrating clozapine, (4) prescribing appropriate antiarrhythmic agents such as verapamil, and (5) conducting electrophysiology studies and radiofrequency ablation if verapamil is not effective. ${ }^{12}$ These measures may reduce the cardiac side-effects of clozapine when used for treatmentresistant schizophrenia.

\section{References}

1. Alvir JM, Lieberman JA, Safferman AZ, Schwimmer JL, Schaaf JA. Clozapine-induced agranulocytosis. Incidence and risk factors in the United States. N Engl J Med 1993;329:162-7. Erossre

2. Freudenreich O, Weiner RD, McEvoy JP. Clozapine-induced electroencephalogram changes as a function of clozapine serum levels. Biol Psychiatry 1997;42:132-7. cross re

3. Merrill DB, Dec GW, Goff DC. Adverse cardiac effects associated with clozapine. J Clin Psychopharmacol 2005;25:32-41. cross ref

4. Sadock BJ. Kaplan and Sadock's Comprehensive Textbook of Psychiatry. 9th ed. Lippincott Williams \& Wilkins; 2009.

5. Orejarena LA, Vidaillet H Jr, DeStefano F, Nordstrom DL, Vierkant RA, Smith PN, et al. Paroxysmal supraventricular tachycardia in the general population. J Am Coll Cardiol 1998;31:150-7. cross re

6. Campbell RW. Supraventricular tachycardia. Occasional nuisance or frequent threat? Eur Heart J 1996;17(Suppl C):21-5. cross ref

7. Kasper DL, Fauci AS, Hauser SL, Longo DL, Jameson JL, Loscalzo J. Harrison's Principles of Internal Medicine. 19th ed. McGraw-Hill, Medical Publishing Division; 2015.

8. Nigro G, Russo V, de Chiara A, Rago A, Cioppa ND, Chianese R, et al. Autonomic nervous system modulation before the onset of sustained atrioventricular nodal reentry tachycardia. Ann Noninvasive Electrocardiol 2010;15:49-55. Eross re

9. Settem JV, Trivedi S, Kamath AG, Behere RV, Kanaradi H, Bhat SM. 
Clozapine-induced supraventricular tachycardia and its treatment with verapamil. Indian J Psychol Med 2015;37:358-9. Eross re

10. Glassman AH, Bigger JT Jr. Antipsychotic drugs: prolonged QTc interval, torsade de pointes, and sudden death. Am J Psychiatry 2001;158:177482. cross re
11. Kilian JG, Kerr K, Lawrence C, Celermajer DS. Myocarditis and cardiomyopathy associated with clozapine. Lancet 1999;354:18415. cross re

12. Ferguson JD, DiMarco JP. Contemporary management of paroxysmal supraventricular tachycardia. Circulation 2003;107:1096-9. Eross ref 